\title{
Are Hadrons Shell-Structured?
}

\author{
Paolo Palazzi \\ particlez.org, PO Box 62, CH-1217 Meyrin 1, Switzerland \\ pp@particlez.org
}

\begin{abstract}
A stability analysis of the mass spectrum indicates that hadrons, like atoms and nuclei, are shell-structured. The mesonic shells mass series, combined with the results of a mass quantization analysis, reveals striking similarities with the nuclear shells. In addition, the mesonic mass patterns suggest solid-phase partonic bound states on an fcc lattice, compatible with a model by A. O. Barut with stable leptons as constituents, bound by magnetism. Baryonic shells grow with a lower density, and only start at shell 3 with the nucleon.
\end{abstract}

Keywords: hadron, shell model; mass, quantization; statistical analysis.

PACS: $12.40 . Y x, 12.90 .+b, 14.40 .-n$.

\section{SHELLS}

Quarks and their mixing are the cornerstone of particle physics, defining an accurate CKM chemistry and a plausible but incomplete taxonomy. The organization of the mass spectrum, however, is far from satisfactory: no comprehensive mass rules are known, and attempts to derive the masses from the quarks and their binding have not succeeded in more than two decades of attempts. Paradoxically, the theory explains a number of experimental results, but cannot compute the most fundamental quantity, energy. The quark model is a set of combinatorial rules for the quantum numbers, but does not offer any structural insight the way atomic or nuclear models do.

In atoms and in nuclei, stability is organized with shells, and to probe the relevance of shells for hadrons, stability can expressed with alignments of the $1 / 3$ power of the total number of constituents of the most stable configurations, the closed shells [1]. To represent the atomic shells, in Fig. 1a the cube root of $Z$ for the inert gases is charted vs. the shell number. Nuclear stability is related mostly to the neutronic magic number series, but with a derived sequence based on the atomic number $A=Z+N$, stability and the spin-orbit coupling effect emerge in a similar way, as shown in Fig. 1b.

Can such patterns be detected in the particle spectrum? Not directly, for lack of an established parton count, but the mass can tentatively be used as a proxy for the number of constituents (which would work for nuclei). By analyzing the distribution of particle lifetimes as a function of the mass, stability peaks are recognized for mesons and for baryons, as shown in Fig. 2a and 2b, and indeed the cube roots of their masses follow two distinct alignments, as shown in Fig. 2c [1]. This would be a strong indication that hadrons are shell-structured if only each constituent contributed a constant amount to the total mass. This assumption is unusual, but not new. 

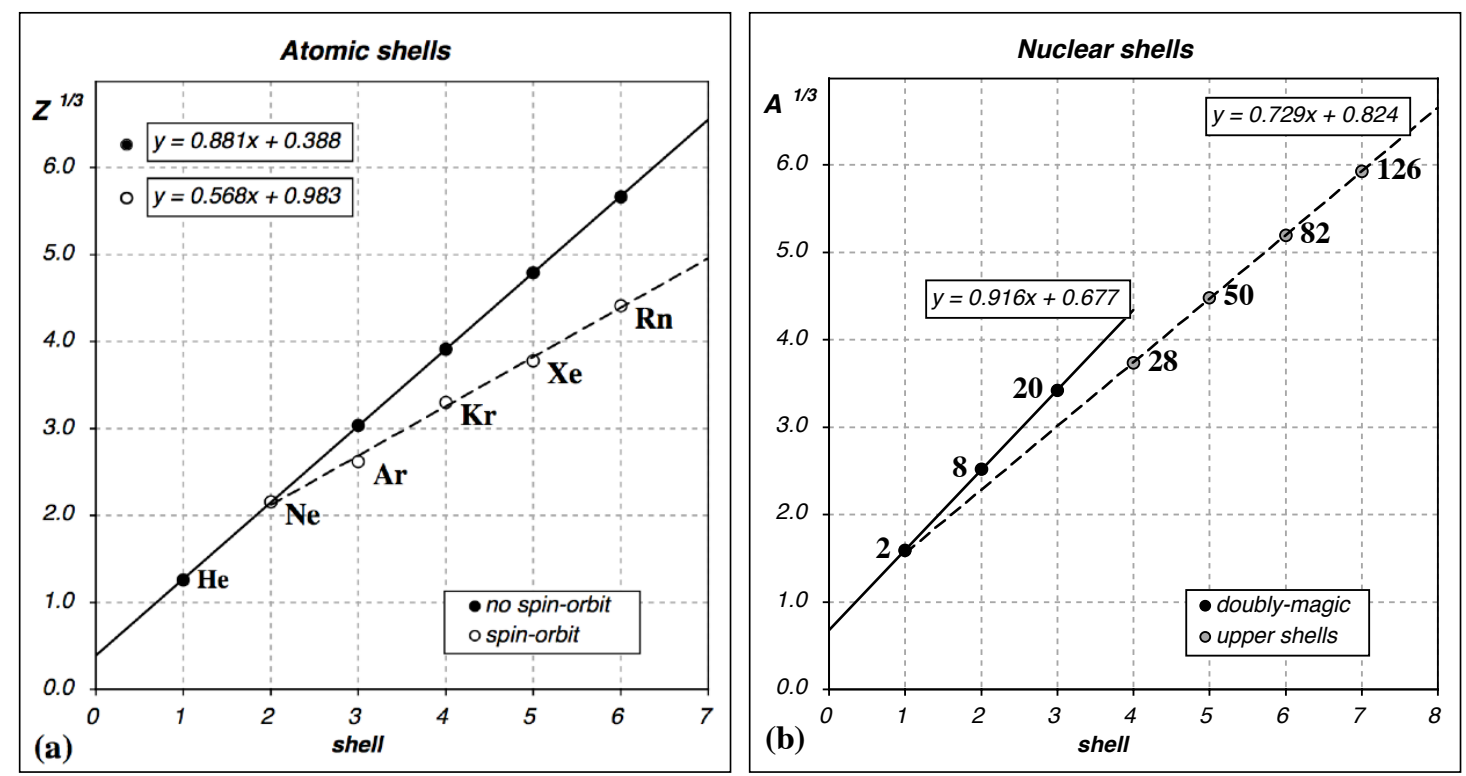

FIGURE 1. a: atomic shells, $Z^{1 / 3}$ vs shell number for the inert gases. The bound state equation identifies the closed shell configurations and determines the parameters of the no spin-orbit line. Spinorbit coupling is effective after shell 2 , and reduces the slope. b: nuclear shells, $A^{\mathbf{1 / 3}}$ vs shell number for the magic neutronic sequence; $A_{\mathrm{i}}=Z_{\mathrm{i}}+N_{\mathrm{i}}$, where $N_{\mathrm{i}}=2,8,20,28, \ldots$ and $Z_{\mathrm{i}}$ is looked up in the Segrè plot along $N_{\mathrm{i}}$ to correspond to maximum stability. After the first three shells, with the doubly-magic nuclei He-4, O-16 and Ca-40, spin-orbit coupling defines a reduced rate of growth (reprinted from [1]).
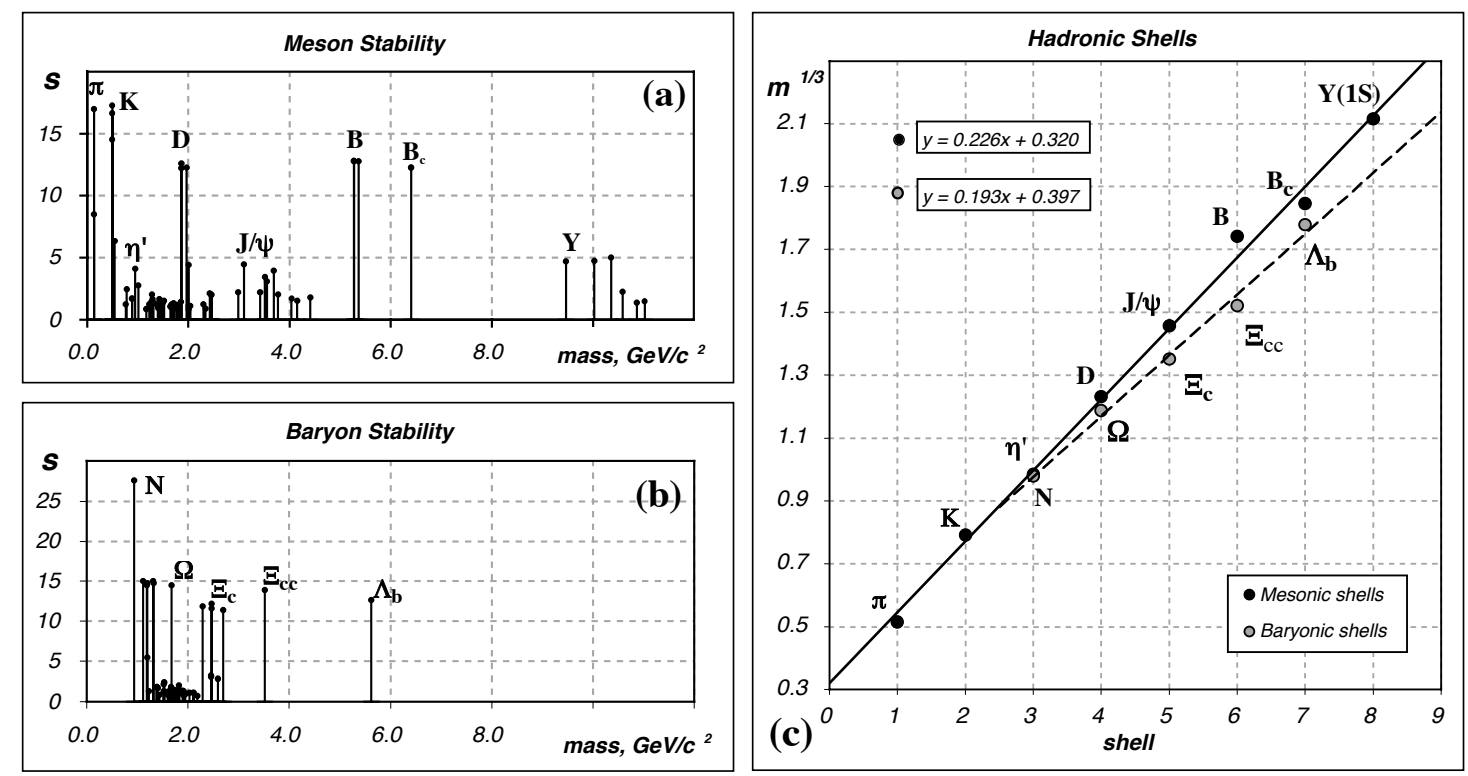

FIGURE 2. a: meson, and b: baryon stability plots showing $s(i)=\lg \left(\tau(i) / \tau\left(Z^{0}\right)\right.$ ) versus the mass (with $i$ running on the whole spectrum), and identification of mesonic shells 1 to 8 and baryonic shells 3 to 7 . c: hadronic shells, expressed as $m^{1 / 3}$ vs. shell number (adapted from [1]). Compared to the mesons, the baryonic shells start only at shell 3, and grow with a lower slope. Deviations from the fitted line are occasionally more important than in the atomic or nuclear shells, and a rationale for this effect is outlined in Ref. [6]. 


\section{MASS QUANTIZATION AND HADRONIC SHELLS}

Y. Nambu observed in 1952 that meson masses are even multiples of a mass unit $u$ of about $35 \mathrm{MeV} / \mathrm{c}^{2}$, baryons (and also unstable leptons) odd multiples, so that mass differences among similar particles are quantized by $70 \mathrm{MeV} / \mathrm{c}^{2}$ [2]. M. H. Mac Gregor studied this property extensively from the '70s to the present time [3] and few other authors mentioned it also. Recently this rule has been reassessed by the present author for all the mesons listed by the PDG, grouped by quark composition and $J^{P C}$, confirming its statistical significance [4]. In addition, the slightly different values of $u$ for the various meson groups, all in the vicinity of $35 \mathrm{MeV} / \mathrm{c}^{2}$, are also quantized on a grid of 12 intervals, and are strongly correlated with the quantum numbers. The baryon mass spectrum shows very similar regularities [5].

The hadronic shells identified by the cube root of mass plot of Fig $2 \mathrm{c}$ can be scaled, one constituent for each mass unit, and re-expressed with $P^{1 / 3}$, where $P$ is the number of partons $P=m / u$ (plots not shown). The mesonic shells thus transformed reveal striking similarities with the nuclear shells: the shell population sequences are almost identical, doubly-magic-equivalent states are present only up to shell 3 , and there are also clear indications of sub-shells [6]. The mesonic shell sequence is correlated with the quark composition up to b-bbar in shell 8, and no states are present around the mass values of the hypothetical subsequent shells. In this context the mesonic mass quantization patterns [4] suggest solid-phase partonic bound states on an fcc lattice, with light spin- $1 / 2$ partons of charge $0,+1$ and -1 with anti-parallel spin coupling and positive binding energy [6]. This interpretation strengthens the mesons-nuclei shell analogy through the fcc nuclear model of N. D. Cook [7].

A possible choice for the constituents, compatible with these results (and the only one with non-fictitious particles), are the stable leptons, as proposed by A. O. Barut, with magnetism acting as the strong interaction among partons [8].

Baryonic shells grow with a lower density compared to the mesons, and start only at shell 3 with the nucleon. The radial charge density distributions of the proton and the neutron [9], as well as the shape of the p-p elastic differential cross-section [10] also indicate that the nucleon is structured in three layers.

\section{REFERENCES}

1. P. Palazzi, "Particles and Shells", http://cdsweb.cern.ch, CERN-OPEN-2003-006 (2003).

2. Y. Nambu, "Empirical Mass Spectrum of Elementary Particles", Prog. Theor. Phys. 7, 595 (1952).

3. M. H. Mac Gregor, "Electron generation of leptons and hadrons with reciprocal alpha-quantized lifetimes and masses", Int. J. Mod. Phys. A20, 719 and 2893 (2005).

4. P. Palazzi, "Patterns in the Meson Mass Spectrum", http://particlez.org, p3a-2004-001 (2004).

5. P. Palazzi, "The Baryon Mass System", in preparation.

6. P. Palazzi, "Meson Shells", http://particlez.org, p3a-2005-001 (2005)

7. N. D. Cook, Models of the Atomic Nucleus, Springer, 2006.

8. A. O. Barut, "Stable Particles as Building Blocks of Matter", Surveys High Energ. Phys. 1, 113 (1980)

9. R. M. Littauer, H. F. Schopper, R. R. Wilson, "Structure of the Proton and Neutron" Phys. Rev. Letters 7, 144 (1961).

10. M. M. Islam, R. J. Luddy and A. V. Produkin, Mod. Phys. Lett., A18, 743 (2003) 